\title{
Efficacy of a New Commercial Ocular Spray Containing Oftasecur Citrus Extract for Reducing Microbial Load in the Conjunctiva of Patients Receiving Intravitreal Injections
}

\author{
Aldo Vagge $\cdot$ Lorenzo Ferro Desideri · Adriano Carnevali (D) C Chiara Del Noce · Davide Camposampiero \\ Marina Agrusta · Diego Ponzin · Marco Pellegrini · Sabrina Vaccaro • Massimo Nicolò • Vincenzo Scorcia • \\ Carlo E. Traverso · Giuseppe Giannaccare
}

Received: July 6, 2021 / Accepted: August 6, 2021 / Published online: September 8, 2021

(C) The Author(s) 2021

\section{ABSTRACT}

Introduction: The control of conjunctival microbial load is crucial for patients receiving intravitreal injections (IVTs) in order to reduce the risk of endophthalmitis. The purpose of this work was to assess the antimicrobial activity of a new commercial ocular spray containing Biosecur citrus extract (Oftasecur ${ }^{\circledR}$, Off Health, Florence, Italy).

Methods: This prospective cross-sectional pilot study included patients receiving IVTs who were instructed to apply Oftasecur spray onto

A. Vagge $\cdot$ L. Ferro Desideri · C. Del Noce .

M. Agrusta - M. Nicolò - C. E. Traverso University Eye Clinic of Genoa, IRCCS Ospedale Policlinico San Martino, Genoa, Italy

A. Vagge · L. Ferro Desideri · C. Del Noce . M. Agrusta - M. Nicolò · C. E. Traverso Department of Neurosciences, Rehabilitation, Ophthalmology, Genetics, Maternal and Child Health (DiNOGMI), University of Genoa, Genoa, Italy

A. Carnevali $(\bowtie) \cdot S$. Vaccaro · V. Scorcia .

G. Giannaccare

Department of Ophthalmology, University Magna

Græcia of Catanzaro, Viale Europa, Germaneto,

88100 Catanzaro, Italy

e-mail: adrianocarnevali@live.it

D. Camposampiero · D. Ponzin

Fondazione Banca degli Occhi del Veneto, Venice, Italy the eye to be injected four times daily starting 4 days before surgery. The contralateral eye was considered the control. A conjunctival swab for microbiological analysis was performed in both eyes before starting study treatment and at the time of the injection. The Brief Ocular Discomfort Inventory (BODI) questionnaire was administered to patients based on an 11-point scale ( 0 for no discomfort and 10 for maximum discomfort).

Results: Thirty patients (15 male, 15 female; mean age $64.7 \pm 11.6$ [standard deviation, SD] years) were included. Before starting treatment, $53.3 \%$ of the total eyes tested positive during

\section{Pellegrini}

Department of Ophthalmology, Ospedali Privati

Forlì "Villa Igea", Forlì, Italy

\section{Pellegrini}

Istituto Internazionale per la Ricerca e Formazione in Oftalmologia (IRFO), Forlì, Italy

M. Pellegrini

Department of Morphology Surgery, and

Experimental Medicine, University of Ferrara, Ferrara, Italy 
the microbiological analysis. After the treatment period, only $20 \%$ of the eyes tested positive at the time of injection, showing a significant reduction in the microbial load $(p<0.01)$. Moreover, in the treated arm, the positive swabs before and after the prophylactic treatment with Oftasecur ocular spray showed a significant reduction (from $70.4 \%$ to $29.6 \%$; $p=0.003$, McNemar's test). Oftasecur ocular spray was well tolerated, with an average BODI score of $1.2( \pm 0.70 \mathrm{SD})$.

Conclusion: Oftasecur ocular spray showed antimicrobial activity that significantly reduced the microbial load in patients receiving intravitreal injections. Therefore, it may have a role in the prophylaxis of infection in the setting of IVTs.

Keywords: Antiseptic; Endophthalmitis prevention; Intravitreal injections; Ocular spray; Oftasecur

\section{Key Summary Points}

Nowadays, treatment with anti-vascular endothelial growth factor (VEGF) drugs through intravitreal injection (IVT) represents the gold standard in the management of age-related macular degeneration.

IVT-related endophthalmitis remains a non-negligible complication, with an estimated incidence ranging from $0.019 \%$ to $0.083 \%$. Prophylaxis with a novel, antiseptic ocular spray composed of bioflavonoids (Oftasecur) may decrease the ocular microbial load of the conjunctiva and potentially further decrease the incidence of IVT-related endophthalmitis.

Oftasecur ocular spray administered four times daily starting 4 days before the IVT procedure is effective in significantly reducing the ocular microbial load of the conjunctiva.
The prophylactic administration of natural and chemical-free antiseptic compounds such as bioflavonoids may represent a viable treatment option in order to decrease the incidence of IVTrelated endophthalmitis, overcoming the drawbacks common to antibiotic agents.

Further larger-scale, randomized clinical trials should provide additional evidence about the role of the topical administration of bioflavonoid antiseptic agents in the prevention of IVT-related endophthalmitis.

\section{INTRODUCTION}

The practice of intravitreal injection (IVT) with anti-vascular endothelial growth factor (VEGF) has significantly impacted the outcomes of exudative macular diseases in recent years and nowadays represents the most frequently performed ocular procedure [1-4]; moreover, the frequency of this procedure is growing worldwide and, in parallel, the risk of procedure-related complications [5]. Among them, endophthalmitis, the most fearful ocular complication, has a reported incidence ranging from $0.019 \%$ to $0.083 \%$ [6-8].

In order to reduce this risk, several preventive treatment options have been investigated [9]; among them, the use of povidone-iodine eye drops in combination with strict antisepsis rules is the only prophylactic modality which has been shown to be effective in reducing endophthalmitis incidence after intraocular surgery [10].

Currently, other ancillary prophylactic agents are still needed in order to further reduce the incidence of endophthalmitis after IVTs. In this regard, flavonoids have been shown to exert direct antioxidant and free radical scavenging activity [9]. Furthermore, these natural compounds display a wide spectrum of biological functions, including antibacterial activity 
against several forms of Gram-negative bacteria [11].

In this context, citrus extract in liposomal form has been described as a natural, non-toxic, chemical-free antiseptic agent, which is composed of citrus bioflavonoids derived from some specific layers of citrus fruits; this natural compound was primarily developed in the food processing industry, because of its effective, broad-spectrum antimicrobial activity both against Gram-negative and some Gram-positive bacteria without the need of adopting nonorganic, synthetic preservatives [12].

Recently, a new commercial ocular spray (Oftasecur $^{\circledR}$, Off Health, Florence, Italy), containing a dispersion of Biosecur liposomes combined with hypromellose and other compounds, has been introduced to the market. Oftasecur ocular spray was developed with the aim of reducing the incidence of severe, infective complications by administering it as a presurgical disinfectant [13].

In this prospective, pilot study we analyzed the antimicrobial activity of Oftasecur ocular spray administered prophylactically in patients receiving IVTs. In addition, we discuss its potential role in preventing possible severe ocular infective complications, including endophthalmitis, as a natural and well-tolerated biocompound.

\section{METHODS}

This multicenter, prospective, cross-sectional, pilot study was performed at the University Eye Clinic of Genoa, Department of Neuroscience, Rehabilitation, Ophthalmology, Genetics, Maternal and Child Health (DINOGMI), IRCCS Ospedale Policlinico San Martino (Genoa, Italy) in collaboration with the Department of Ophthalmology, University Magna Græcia of Catanzaro (Catanzaro, Italy). The International Center for Ocular Physiopathology, Fondazione Banca degli Occhi del Veneto Onlus (Venice, Italy) was involved in the microbiological analysis. Informed consent was acquired from all participants, and the study was carried out in accordance with the Declaration of Helsinki, with approval obtained by the local institutional ethics committees.

In this study, patients receiving IVTs in one eye were included. The eye undergoing IVT was considered the study eye, while the fellow eye was considered the control eye. Patients were instructed to apply Oftasecur ocular spray four times daily (at $8.00 \mathrm{am}, 12.00 \mathrm{pm}, 4.00 \mathrm{pm}$, $8.00 \mathrm{pm}$ ), starting 4 days before the intraocular procedure (T0), onto the eye to be injected. A conjunctival swab was performed in both eyes at T0 and repeated at the time of the IVT (T1), after the 4-day treatment period with Oftasecur ocular spray. The Brief Ocular Discomfort Inventory (BODI) questionnaire was administered to all the patients, based on an 11-point scale (0 for no discomfort and 10 for discomfort as severe as you can imagine) [14].

All the conjunctival swab samples were sent to the International Center for Ocular Physiopathology, Fondazione Banca degli Occhi del Veneto Onlus (Venice, Italy) for the microbiological analysis. To ensure growth of microorganisms, samples were incubated at $37^{\circ} \mathrm{C}$ in a tube of thioglycollate broth for $24 \mathrm{~h}$ after the collection.

Statistical analysis was performed using SPSS for Windows (SPSS Inc., Chicago, IL, USA). The parameters of the two groups were compared using Student's $t$ test for continuous variables. McNemar's test was performed to compare paired nominal data. A $p$-value $<0.05$ was considered statistically significant, and all $p$ values were based on two-tailed tests.

\section{RESULTS}

Overall, 60 eyes of 30 patients (15 males, 15 females; mean age $64.7 \pm 11.6$ [standard deviation, SD]) receiving IVTs were included in this study. The 30 injected eyes were included in the study group, while the 30 fellow eyes were considered the controls. In total, $56.7 \%$ of the patients $(n=17 / 30)$ were treated for diabetic macular edema, $23.3 \%(n=7 / 30)$ for wet agerelated macular degeneration, $13.3 \%(n=4 / 30)$ for retinal vein occlusion and the remaining $6.7 \%(n=2 / 30)$ for myopic choroidal neovascularization. Before starting treatment, 53.3\% 
( $n=16 / 30)$ of the eyes tested positive after the microbiological analysis. The microbial load decreased significantly after the prophylactic treatment, and only $20 \%$ of the eyes $(n=17 / 30)$ tested positive at the time of injection $(p<0.01)$.

In treated eyes, there was a statistically significant difference in the proportion of positive swabs before and after the prophylactic treatment with Oftasecur (from $70.4 \%$ to $29.6 \%$, $p=0.003$, McNemar's test). By contrast, in
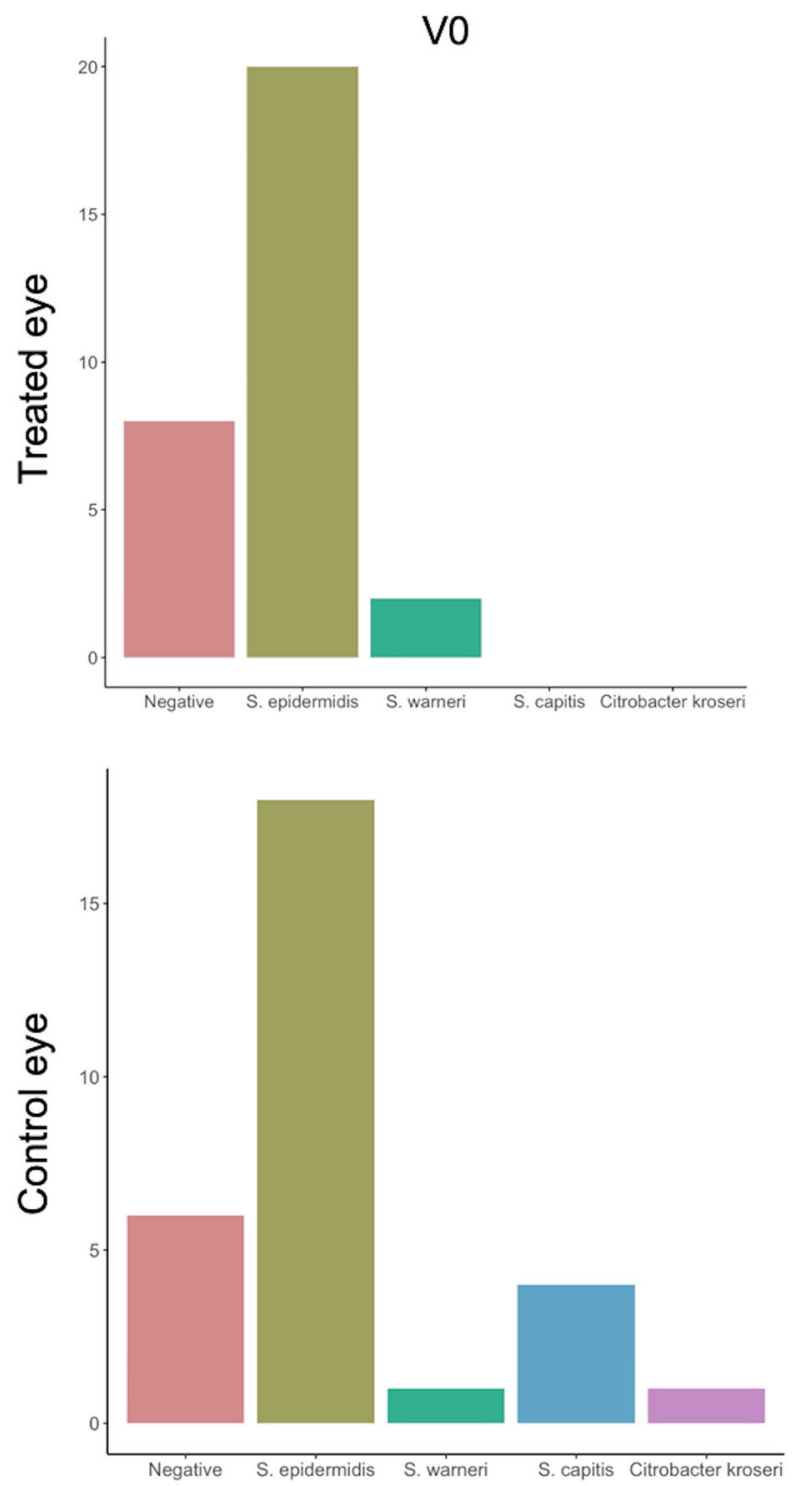

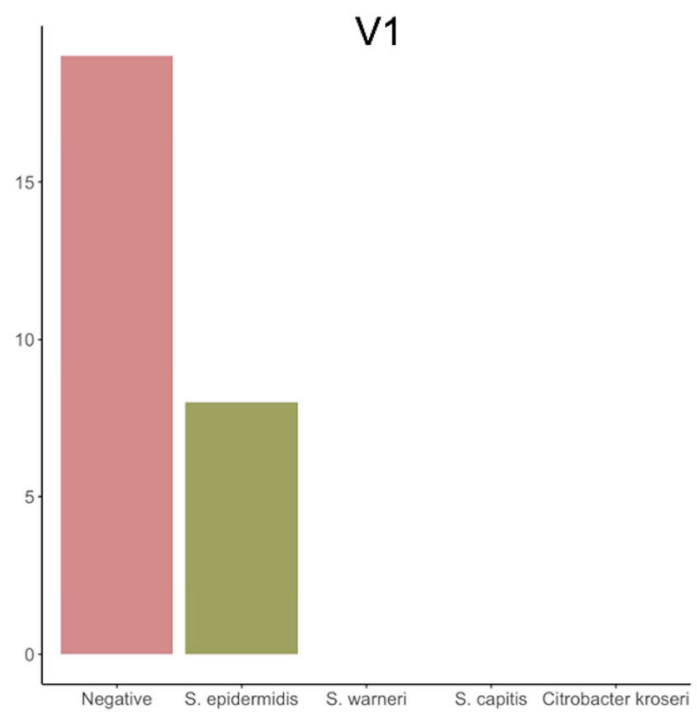

control eyes, the number of positive swabs did not change significantly (from $81.5 \%$ to $55.6 \%$, $p=0.070$, McNemar's test). The different microorganisms cultured from conjunctival swabs and their percentages are summarized below (Fig. 1). No injected eyes showed any clinical sign of infection during the postoperative evaluation.

Oftasecur ocular spray was well tolerated by all the subjects in the study group; moreover, no subject complained of ocular discomfort,

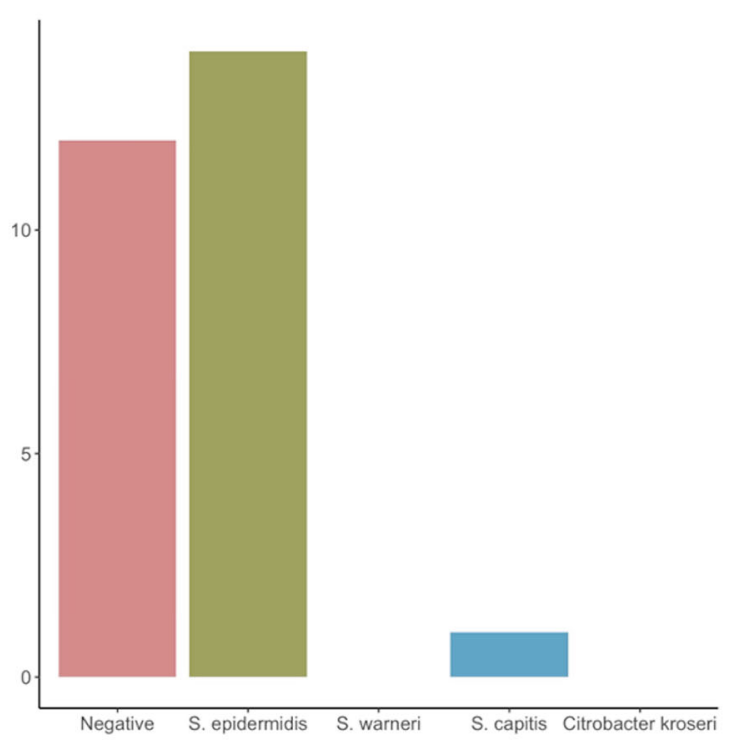

Fig. 1 Different isolated bacterial species before and after application of Oftasecur ocular spray prophylaxis in treated and control eyes 
and all the study subjects adhered to the prophylactic treatment schedule. The average BODI score was $1.2( \pm 0.70 \mathrm{SD})$.

\section{DISCUSSION}

Postoperative endophthalmitis remains one the most feared complications following intraocular procedures such as IVTs. In fact, it is often associated with poor visual outcomes and in some cases may cause complete vision loss [6].

The most frequently isolated etiologic agents in postoperative endophthalmitis are represented by Gram-positive organisms, accounting for $95 \%$ of culture-positive isolates [15]. In particular, most of the reported Gram-positive bacteria (around 70\%) are coagulase-negative micrococci (including Staphylococcus epidermidis) and, less commonly, Staphylococcus aureus (10\%), Streptococcus species (9\%) and Enterococcus species (2.2\%). Conversely, Gram-negative species account for about 5-6\% of cultures and include Pseudomonas, Proteus, and Haemophilus influenzae [16].

Considering the non-negligible incidence and the clinical sequelae of IVT-related endophthalmitis, its prevention remains a primary endpoint in the management of patients treated with IVTs. From this perspective, although prophylactic treatment with povidone-iodine eye drops has reduced the incidence of endophthalmitis after intraocular surgery, ancillary methods are still needed to further reduce the risk [10].

In this prospective, cross-sectional, pilot study, we analyzed the efficacy of Oftasecur ocular spray in reducing the microbial load in patients undergoing IVTs. We found a significant decrease in the microbial load in patients treated with Oftasecur after the prophylactic treatment, while no difference was detected in control non-treated eyes. Moreover, in line with the abovementioned literature, most of the isolated microorganisms were Gram-positive coagulase-negative micrococci, (in particular Staphylococcus epidermidis), which in most of the post-treatment swabs tested negative as opposed to controls. Oftasecur showed a promising broad-spectrum activity, against both Gram-positive and Gram-negative microorganisms. In this regard, some previous in vitro studies have evaluated the prophylactic antibacterial activity of ophthalmic drops containing povidone-iodine. Musumeci et al. compared the antimicrobial activity between $0.6 \%$ povidone-iodine and $5 \%$ povidone-iodine formulations in vitro. They found that the $0.6 \%$ povidone-iodine formulation was faster than the $5 \%$ povidone-iodine preparation in reducing the Gram-positive (including Staphylococcus epidermidis) and Gram-negative bacterial load [17]. Another preclinical study confirmed the antimicrobial activity of $0.6 \%$ povidone-iodine ophthalmic solution, which was effective in neutralizing in vitro growth of Staphylococcus epidermidis, Staphylococcus aureus, Pseudomonas aeruginosa and Candida species [18]. Currently, the only prophylactic ophthalmic solution tested in clinical studies is $5 \%$ povidone-iodine preparation. A prospective study on 24 patients undergoing IVTs reported that the irrigation of conjunctival fornix with $5 \%$ povidone-iodine was more effective in reducing the microbial load as compared with drop application of the same solution, probably due to the longer exposure of the conjunctiva to the drug [19]. Other studies have investigated the potential additive bactericidal role of antibiotic drops a few days before IVTs; however, their clinical efficacy in comparison with povidone-iodine alone is still controversial [20-22].

In recent years the role of antibiotic resistance has become an important issue, not only in ophthalmology but in all fields of medicine. In fact, the routine adoption of antibiotics for infection prophylaxis during multiple IVT courses has been associated with an increased risk of developing antibiotic resistance in the conjunctival flora $[23,24]$.

For example, a randomized clinical study demonstrated that coagulase-negative staphylococci (CNS) cultured from eyes repeatedly treated with fluoroquinolones developed significantly higher rates of resistance to this class of antibiotics [25]. Similarly, antibiotic resistance has been reported with repeated treatment courses with macrolides (for example azithromycin) [26]. Moreover, sustained administration of antibiotics may lead to 
changes in the normal conjunctival flora along with an increased percentage of Staphylococcus epidermidis [27].

For these reasons, antiseptic agents, including povidone-iodine or biguanides, are being investigated as a viable alternative in the prophylaxis of IVT-related infectious complications. Indeed, these agents have shown comparable efficacy to topical antibiotics without the risk of developing antibiotic resistance [28]. In this direction, Oftasecur ocular spray may represent a promising prophylactic treatment option in reducing the risk of postoperative endophthalmitis, without altering the normal conjunctival flora composition. Oftasecur belongs to the class of bioflavonoids, which act as antioxidants and free radical scavengers, and have the capability of exerting enzymatic activity and inhibiting cell proliferation [29]. In this regard, an in vitro study investigated the antimicrobial activity (against Vibrio vulnificus) of Biosecur at two different concentrations $(0.5 \%$ and $2 \%)$. Biosecur at $2 \%$ revealed similar antimicrobial activity as tetracyclines. Furthermore, Biosecur at $2 \%$ led to a $6-\log$ reduction in Vibrio vulnificus and at $0.5 \%$ to a $3-\log$ reduction in the bacillus. Biosecur at $2 \%$ showed residual activity at least $2.5 \mathrm{~h}$ after application. Results from this in vitro study suggest that Biosecur exhibits a high level of antimicrobial activity (similar to tetracyclines), with long-lasting residual effects, and its safe and organic profile represents another important advantage over nonorganic and possibly more toxic products [12].

In clinical practice, the formulation of Oftasecur as an ocular spray works by delivering the molecule droplets to the eyelid margin, allowing the patient to mix the drug with the tear film while opening the eyes. This ocular delivery system has been shown to be more effective than traditional eye drops in the case of physical incapacity, previous ocular diseases or low patient compliance [30]. Thus, Oftasecur ocular spray may display an additional advantage over traditional eye drops in older and less compliant patients, which commonly represent the majority of individuals undergoing IVTs.

This study has some limitations, including the relatively small sample size considering its nature as a pilot study and the non-randomized design. A large randomized study is currently underway at our institutions in order to further validate these results. Another limitation is that the sensitivity profile of the isolated microorganisms was not examined. Further studies should also investigate this important issue.

\section{CONCLUSIONS}

In conclusion, Oftasecur ocular spray may represent a viable, non-toxic, ancillary preoperative option for patients undergoing IVTs, given its efficacy in decreasing the microbial load and thus the risk of IVT-related endophthalmitis. Further larger-scale studies should provide more data about its clinical efficacy in reducing the risk of IVT-related endophthalmitis.

\section{ACKNOWLEDGEMENTS}

Funding. No funding or sponsorship was received for this study or publication of this article.

Authorship. All named authors meet the International Committee of Medical Journal Editors (ICMJE) criteria for authorship for this article, take responsibility for the integrity of the work as a whole, and have given their approval for this version to be published.

Authorship Contributions. All authors contributed to the study conception and design. Material preparation, data collection and analysis were performed by Lorenzo Ferro Desideri, Chiara Del Noce, Davide Camposampiero, Marina Agrusta, Diego Ponzin, Aldo Vagge, Adriano Carnevali, Sabrina Vaccaro, Giuseppe Giannaccare. The first draft of the manuscript was written by Aldo Vagge and Lorenzo Ferro Desideri. All authors commented on previous versions of the manuscript. All authors read and approved the final manuscript.

Disclosures. Aldo Vagge, Lorenzo Ferro Desideri, Adriano Carnevali, Chiari Del Noce, 
Davide Camposampiero, Marina Agrusta, Diego Ponzin, Marco Pelligrini, Sabrina Vaccaro, Massimo Nicolò, Vincenzo Scorcia, Carlo Traverso and Guiseppe Giannaccare all confirm that they have no conflicts of interest to declare.

Compliance with Ethics Guidelines. Informed consent was acquired from all the participants, and the study was carried out in accordance with the Declaration of Helsinki, with approval by the local institutional ethics committees (Comitato etico Regione Calabria, protocol number: 312. Date of approval: 17/09/ 2020).

Data Availability. Data sharing is not applicable to this article as no datasets were generated or analyzed during the current study.

Open Access. This article is licensed under a Creative Commons Attribution-NonCommercial 4.0 International License, which permits any non-commercial use, sharing, adaptation, distribution and reproduction in any medium or format, as long as you give appropriate credit to the original author(s) and the source, provide a link to the Creative Commons licence, and indicate if changes were made. The images or other third party material in this article are included in the article's Creative Commons licence, unless indicated otherwise in a credit line to the material. If material is not included in the article's Creative Commons licence and your intended use is not permitted by statutory regulation or exceeds the permitted use, you will need to obtain permission directly from the copyright holder. To view a copy of this licence, visit http://creativecommons.org/licenses/by$\mathrm{nc} / 4.0 /$.

\section{REFERENCES}

1. Lanzetta P, Mitchell P, Wolf S, Veritti D. Different antivascular endothelial growth factor treatments and regimens and their outcomes in neovascular age-related macular degeneration: a literature review. Br J Ophthalmol. 2013;97:1497-507.
2. Nicolo M, Morlacchi A, Cappelli F, et al. Real-life data in the treatment of neovascular age-related macular degeneration: results from the Imaculaweb Registry evaluated in a single Italian Medical Retina Center. Ophthalmologica. 2020;243(6):453-60.

3. Ferro Desideri L, Traverso CE, Nicolo M. Brolucizumab: a novel anti-VEGF humanized singlechain antibody fragment for treating w-AMD. Expert Opin Biol Ther. 2021;21:553-61.

4. Nicolo M, Ferro Desideri L, Vagge A, Traverso CE. Faricimab: an investigational agent targeting the Tie-2/angiopoietin pathway and VEGF-A for the treatment of retinal diseases. Expert Opin Investig Drugs. 2021;30(3):193-200.

5. Lau PE, Jenkins KS, Layton CJ. Current evidence for the prevention of endophthalmitis in anti-VEGF intravitreal injections. J Ophthalmol. 2018;2018: 8567912.

6. Fileta JB, Scott IU, Flynn HW Jr. Meta-analysis of infectious endophthalmitis after intravitreal injection of anti-vascular endothelial growth factor agents. Ophthalmic Surg Lasers Imaging Retina. 2014;45:143-9.

7. Bhavsar AR, Googe JM Jr, Stockdale CR, et al. Risk of endophthalmitis after intravitreal drug injection when topical antibiotics are not required: the diabetic retinopathy clinical research network laserranibizumab-triamcinolone clinical trials. Arch Ophthalmol. 2009;127:1581-3.

8. Englander M, Chen TC, Paschalis EI, Miller JW, Kim IK. Intravitreal injections at the Massachusetts Eye and Ear Infirmary: analysis of treatment indications and postinjection endophthalmitis rates. $\mathrm{Br} \mathrm{J}$ Ophthalmol. 2013;97:460-5.

9. Torres-Costa S, Ramos D, Brandao E, et al. Incidence of endophthalmitis after intravitreal injection with and without topical antibiotic prophylaxis. Eur J Ophthalmol. 2020. https://doi.org/10.1177/ 1120672120902028.

10. Peden MC, Hammer ME, Suner IJ. Dilute povidoneiodine prophylaxis maintains safety while improving patient comfort after intravitreal injections. Retina. 2019;39(11):2219-24.

11. Burt S. Essential oils: their antibacterial properties and potential applications in foods-a review. Int J Food Microbiol. 2004;94:223-53.

12. Cormier J, Scott R, Janes M. Antibacterial activity of biosecur((R)) citrus extract surface cleaner against Vibrio vulnificus. Open Microbiol J. 2013;7:130-4. 
13. Health O. Oftasecur ocular spray. 2020. https:// www.offhealth.it/notizie/oftasecur-sicurezzaoftalmologia/.

14. Sayegh RR, Yu Y, Farrar JT, et al. Ocular discomfort and quality of life among patients in the dry eye assessment and management study. Cornea. 2021;1(40):869-76.

15. Results of the Endophthalmitis Vitrectomy Study. A randomized trial of immediate vitrectomy and of intravenous antibiotics for the treatment of postoperative bacterial endophthalmitis. Endophthalmitis Vitrectomy Study Group. Arch Ophthalmol. 1995;113:1479-96.

16. Wu L, Berrocal MH, Arevalo JF, et al. Endophthalmitis after pars plana vitrectomy: results of the Pan American Collaborative Retina Study Group. Retina. 2011;31:673-8.

17. Musumeci R, Bandello F, Martinelli M, Calaresu E, Cocuzza CE. In vitro bactericidal activity of $0.6 \%$ povidone-iodine eye drops formulation. Eur J Ophthalmol. 2019;29:673-7.

18. Pinna A, Donadu MG, Usai D, et al. In vitro antimicrobial activity of a new ophthalmic solution containing povidone-iodine $0.6 \%$ (IODIM $((\mathrm{R})))$. Acta Ophthalmol. 2020;98:e178-80.

19. Safar A, Dellimore MC. The effect of povidone iodine flush versus drops on conjunctival colonization before intravitreal injections. Int Ophthalmol. 2007;27:307-12.

20. Moss JM, Sanislo SR, Ta CN. A prospective randomized evaluation of topical gatifloxacin on conjunctival flora in patients undergoing intravitreal injections. Ophthalmology. 2009;116:1498-501.

21. Coskun M, Altintas AG, Anayol MA, Raza S, Celikbilek N, Simsek S. Evaluation of efficacy of topical povidone-iodine and different types of fluoroquinolones in the sterilization of bacterial flora on the conjunctiva. J Ocul Pharmacol Ther. 2011;27: 589-92.
22. Li B, Nentwich MM, Hoffmann LE, et al. Comparison of the efficacy of povidone-iodine $1.0 \%, 5.0 \%$, and $10.0 \%$ irrigation combined with topical levofloxacin $0.3 \%$ as preoperative prophylaxis in cataract surgery. J Cataract Refract Surg. 2013;39: 994-1001.

23. Budzinskaya MV, Khalatyan AS, Strakhovskaya MG, Zhukhovitsky VG. Ocular flora in patients undergoing intravitreal injections: antibiotic resistance patterns and susceptibility to antiseptic picloxydine. Int J Ophthalmol. 2020;13:85-92.

24. Grzybowski A, Brona P, Kim SJ. Microbial flora and resistance in ophthalmology: a review. Graefes Arch Clin Exp Ophthalmol. 2017;255:851-62.

25. Kim SJ, Toma HS. Antimicrobial resistance and ophthalmic antibiotics: 1-year results of a longitudinal controlled study of patients undergoing intravitreal injections. Arch Ophthalmol. 2011;129: 1180-8.

26. Dave SB, Toma HS, Kim SJ. Ophthalmic antibiotic use and multidrug-resistant staphylococcus epidermidis: a controlled, longitudinal study. Ophthalmology. 2011;118:2035-40.

27. Dave SB, Toma HS, Kim SJ. Changes in ocular flora in eyes exposed to ophthalmic antibiotics. Ophthalmology. 2013;120:937-41.

28. Barkana Y, Almer Z, Segal O, Lazarovitch Z, Avni I, Zadok D. Reduction of conjunctival bacterial flora by povidone-iodine, ofloxacin and chlorhexidine in an outpatient setting. Acta Ophthalmol Scand. 2005;83:360-3.

29. Duthie G, Crozier A. Plant-derived phenolic antioxidants. Curr Opin Lipidol. 2000;11:43-7.

30. Vagge A, Del Noce C, Pellegrini M, et al. Efficacy of a cycloplegic agent administered as a spray in the pediatric population. J Pediatr Ophthalmol Strabismus. 2020;57:301-4. 